\title{
On the Mechanism of Electrochemical Generation and Decomposition of Phthalimide $\mathbf{N}$-oxyl (PINO)
}

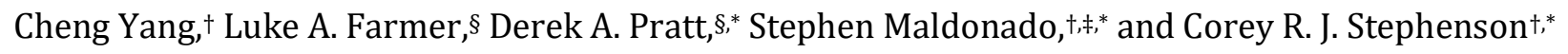 \\ † Willard Henry Dow Laboratory, Department of Chemistry, University of Michigan, 930 North University Avenue, Ann Arbor, Mich- \\ igan 48109, United States. \\ ‡ Program in Applied Physics, University of Michigan, Ann Arbor, Michigan 48109, United States. \\ $\S$ Department of Chemistry and Biomolecular Sciences, University of Ottawa, Ottawa, ON K1N 6N5, Canada. \\ *Email: dpratt@uottawa.ca; smald@umich.edu and crjsteph@umich.edu.
}

Supporting Information Placeholder

\begin{abstract}
Phthalimide $N$-oxyl (PINO) is a potent hydrogen atom transfer (HAT) catalyst that can be generated electrochemically from $\mathrm{N}$-hydroxyphthalimide (NHPI). However, catalyst decomposition has limited its application. This paper details mechanistic studies of the generation and decomposition of PINO under electrochemical conditions. Voltammetric data, observations from bulk electrolysis, and computational studies suggest two pri-

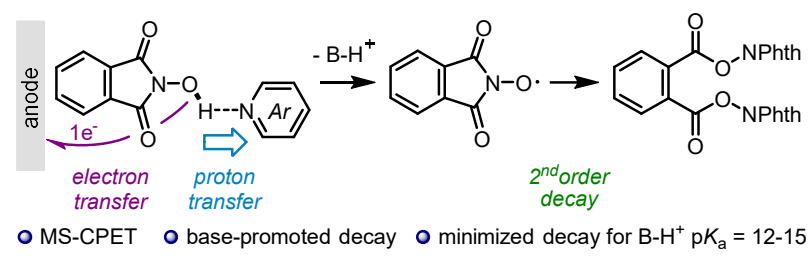
mary aspects. First, base-promoted formation of PINO from NHPI occurs via multiple-site concerted proton-electron transfer (MS-CPET). Second, PINO decomposition occurs by at least two second-order paths, one of which is greatly enhanced by base. Optimal catalytic efficiency in PINO-catalyzed oxidations occurs in the presence of bases whose corresponding conjugate acids have $\mathrm{p} K_{\mathrm{a}} \mathrm{s}$ in the range of 12-15, which strike a balance between promoting PINO formation and minimizing its decay.
\end{abstract}

\section{- INTRODUCTION}

Electrocatalysts that promote anodic half reactions have been specifically developed for oxidative organic transformations due to longstanding industrial interest. ${ }^{1}$ The use of oxidative electrocatalysts, shuttling electrons between molecular substrates and anode interfaces, have realized chemical transfromations in an environmentally friendly manner, as electrons are collected by electrode interfaces instead of stoichiometric chemical oxidants. ${ }^{2}$ For example, the electrochemically-generated $N$-oxoammonium ion $\left(\mathrm{TEMPO}^{+}\right)$derived from TEMPO serves as an active catalytic species and readily reacts with alcohols to produce ketones. ${ }^{3}$ Both TEMPO and $\mathrm{TEMPO}^{+}$salt are isolable, which facilitates mechanistically-guided development of this and related redox mediators for selective and efficient oxidations. ${ }^{3}$

PINO is another promising electrocatalyst generated by the facile oxidation of its precursor NHPI (Figure 1A). ${ }^{3}$ PINO can abstract allylic ${ }^{4}$ and benzylic ${ }^{5-7}$ hydrogen atoms, as well as other relatively weak $\mathrm{C}$-H bonds, ${ }^{8}$ making it a potent HAT electrocatalyst. However, PINO is short-lived, which has caused a limited operational utility for methodology development due to PINO decomposition. ${ }^{8}$ The practical consequence of this decomposition is the need to use NHPI at high catalyst loading (10-20 mol\%) to achieve efficient reactivity, as exemplified in the application to the oxidation of $\beta-0$ 4 linkages in lignin. ${ }^{6}$ Furthermore, the short lifetime of PINO has made its mechanistic studies challenging. ${ }^{3}$ Despite the interest in the NHPI/PINO couple as a valuable redox mediator, ${ }^{3}$ a full assessment of off-cycle (i.e., PINO decomposition) pathways has not been performed.

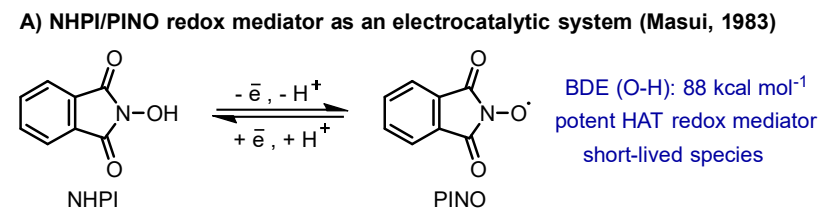

B) PINO decompostion (Masui, 1987)
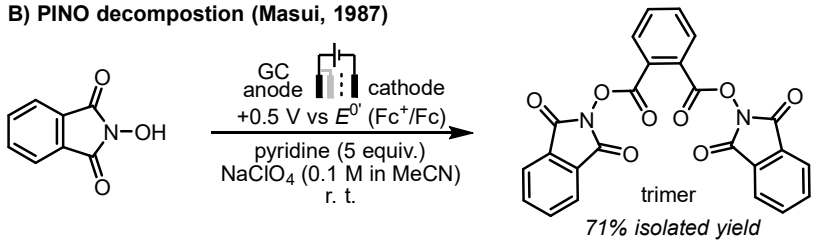

C) Previously proposed decomposition mechanisms

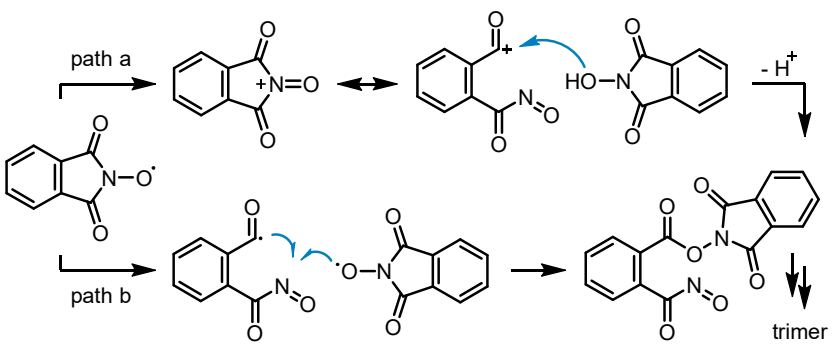

Figure 1. Previous studies on PINO decomposition under electrochemical conditions.

PINO decomposition under electrochemical conditions was first reported by Masui and co-workers in $1987 .{ }^{9} \mathrm{~A}$ trimeric species (hereafter trimer) was isolated and characterized as the major PINO decomposition product after bulk electrolysis of NHPI in the presence of pyridine (Figure 1B). 

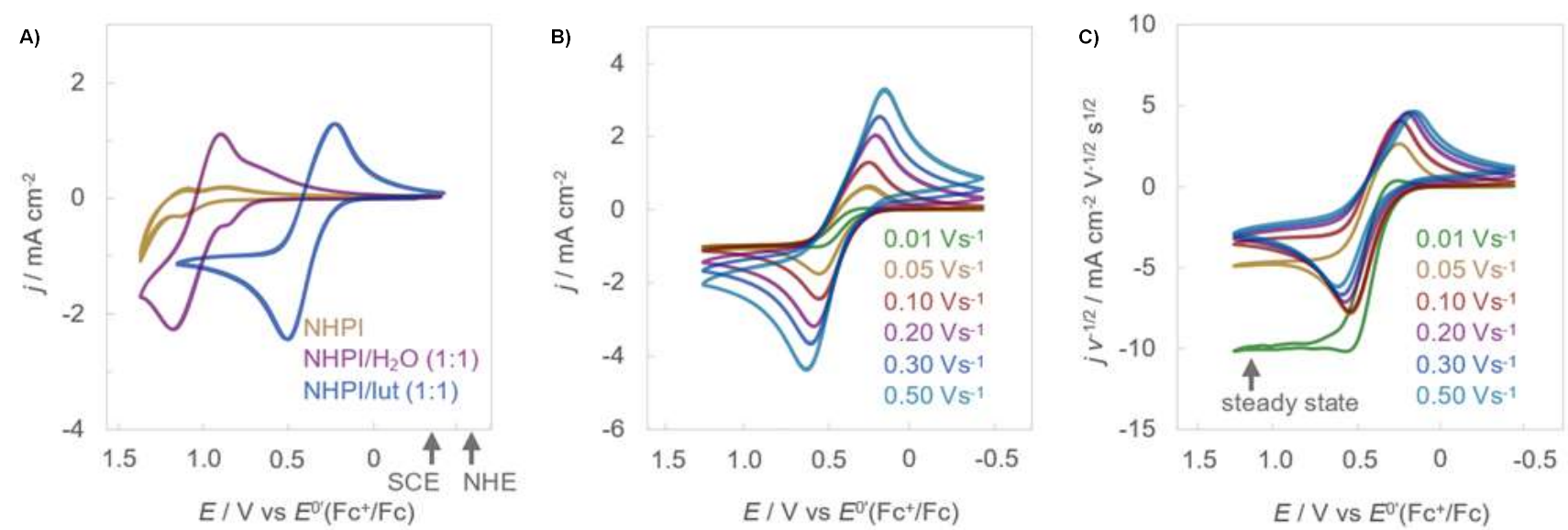

Figure 2. A) Cyclic voltammograms for oxidation of $10 \mathrm{mM}$ NHPI (yellow); and in the presence of $10 \mathrm{mM}$ water (purple); in the presence of $10 \mathrm{mM}$ 2,6-lutidine (blue) at the scan rate of $0.1 \mathrm{Vs}^{-1}$. B and C) Cyclic voltammograms for oxidation of $10 \mathrm{mM}$ NHPI in the presence of $10 \mathrm{mM}$ 2,6-lutidine at the scan rate varying from $0.01 \mathrm{Vs}^{-1}$ to $0.1 \mathrm{Vs}^{-1}$.

The formation of the trimer by nucleophilic attack of NHPI upon its oxoammonium ion was suggested (Figure 1C, path a). Later, Pedulli and co-workers proposed a separate radical pathway involving coupling between PINO and the acyl radical arising from $\mathrm{C}-\mathrm{N}$ bond fragmentation of another PINO (Figure 1C, path b). ${ }^{10}$ Both decomposition pathways have been presumed as relevant in the literature without detailed scrutinity. Advancing the NHPI/PINO redox mediator as an efficient catalytic system requires disambiguation of the PINO decomposition mechanisms.

To enable development of new strategies for selective electrochemical oxidation using NHPI or similar species, we have examined elementary aspects of PINO generation, and ascertained how it participates in oxidation of benzylic alcohols using electroanalytical and computational methods. The data indicate a multiple-site concerted proton-electron transfer (MS-CPET) mechanism of PINO generation from NHPI, where the proton and electron move to different locations. ${ }^{11}$ Moreover, it suggests that in lieu of the two aforementioned mechanistic proposals for the decomposition of PINO, electrochemically-generated PINO undergoes two distinct second-order decay processes, one of which is promoted by base. The influence of base strength on the catalytic efficiency of benzylic alcohol oxidation is quantitively analyzed. Taken together, this study provides a foundation for the development of more effective $N$-oxyl electrocatalysts.

\section{- RESULTS}

Methods and Materials. The study was conducted in the sequence of analyzing the redox response of NHPI with and without bases, then NHPI-base complex, and ascertaining the base activity on substrate oxidation, finally simulating PINO decomposition mechanisms computationally. All experiments were performed in anhydrous acetonitrile (MeCN) due to its high dielectric constant, inertness towards NHPI and PINO, and comparatively high solubility of NHPI relative to other solvents. The energetics associated with NHPI complexation, electrochemical oxidation and possible mechanisms of PINO decomposition were assessed using density functional theory. Unless otherwise indicated, the calculations were carried out using the B3LYP functional with $6-311+G(d)$ basis set and implicit solvation (IEFPCM) parameterized for MeCN.

Redox Response of NHPI with/without Bases. The redox response of NHPI at a glassy carbon electrode was measured in anhydrous MeCN with recrystallized tetrabutylammonium hexafluorophosphate $\left(\mathrm{NBu}_{4} \mathrm{PF}_{6}\right){ }^{12}$ Figure $2 \mathrm{~A}$ shows the voltammetric response of NHPI without base in the electrolyte. In contrast to numerous previous reports, including our own, ${ }^{4-7,13-14}$ a reversible voltammetric response was not observed. Instead, NHPI could not be readily oxidized at 0.1 $\mathrm{Vs}^{-1}$ in this potential range under anhydrous conditions. Moreover, the lack of cathodic current on the return sweep suggested oxidized NHPI $\left(\mathrm{NHPI}^{\bullet}\right)$ ) was unstable on this timescale. The redox response of NHPI oxidation speaks aganist a common observation that NHPI can be reversibly oxidized both with and without a Brønsted base. ${ }^{4-7,13-14}$

Variation in the electrolyte did not affect the voltammetric response for NHPI unless care was not taken to exclude water (Supporting Information, SI). Adventitious and/or intentional addition of water to the electrolyte caused the voltammetric response to appear quasi-reversible, implicating water as an active agent during the oxidation of NHPI and suggesting similar wet conditions were likely used in prior reports of electrochemical oxidation of NHPI without

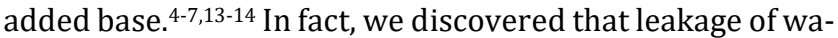
ter $\left(\mathrm{H}_{2} \mathrm{O}\right)$ from an aqueous reference electrode was sufficient to cause water contamination. The formal standard potential for NHPI oxidation in the presence of added $\mathrm{H}_{2} \mathrm{O}$, as determined from the midpoint of the redox waves $\left(E^{0^{\prime}} \approx\right.$ $E_{1 / 2}$ ), was a function of the concentration of added water (SI). When one equivalent of water was used, $E_{1 / 2}=+1.04 \mathrm{~V}$ vs $E^{0^{\prime}}\left(\mathrm{Fc}^{+} / \mathrm{Fc}\right.$ ) (Figure 2A). ${ }^{15}$

Cyclic voltammetric responses for the oxidation of NHPI in the presence of 1 equivalent of 2,6-lutidine were collected from $0.01 \mathrm{Vs}^{-1}$ to $0.5 \mathrm{Vs}^{-1}$ (Figure $2 \mathrm{~B}$ ). Normalizing the current densities in Figure 2C by $\sqrt{v}$ highlighted the fact that the voltammetric response shape was sensitive to the experimental timescale, indicating the oxidation of NHPI was coupled with at least one additional chemical reaction. 

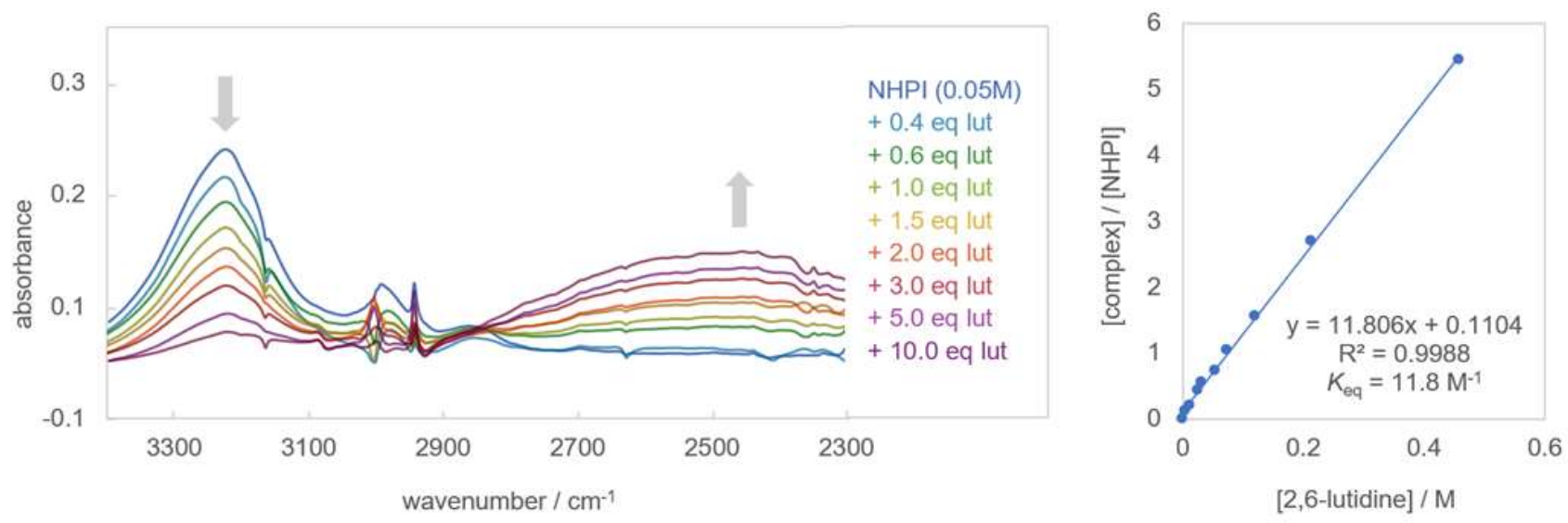

B) Cyclic voltammetric titration of NHPI with 2,6-lutidine

C) Computations of $\mathrm{NHPI} / \mathrm{base}$ interactions
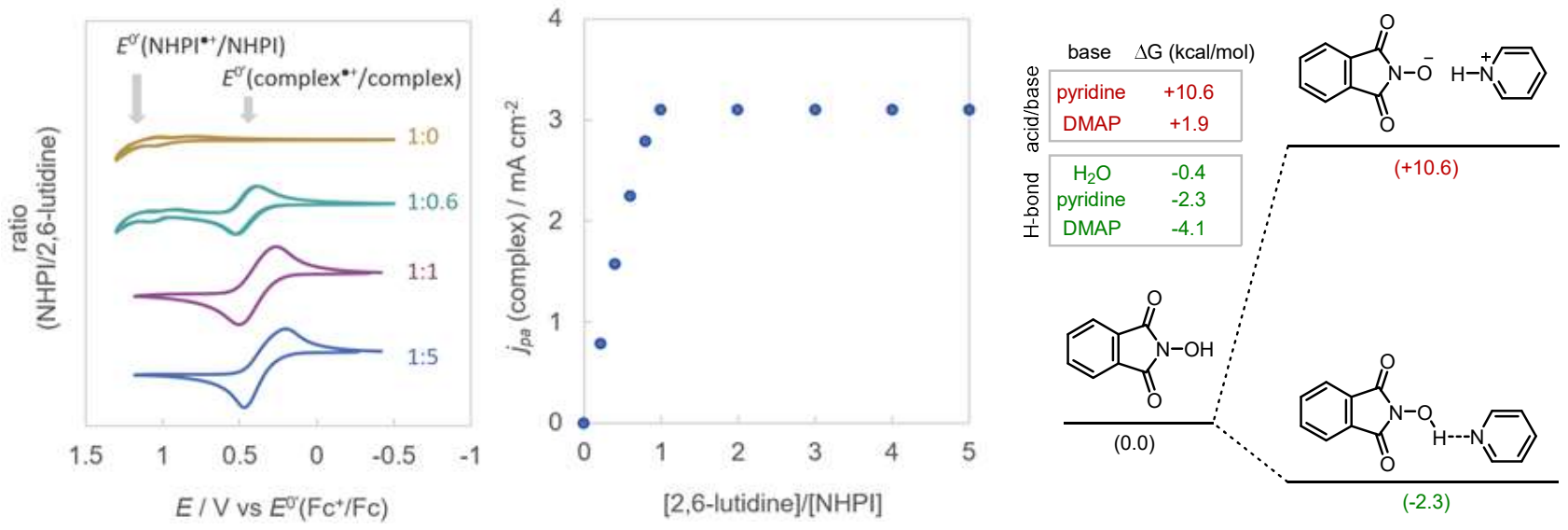

Figure 3. A) IR spectroscopy of 0.05 M NHPI in MeCN, followed by addition of 2,6-lutidine. B) Selected cyclic voltammograms for titration of $10 \mathrm{mM}$ NHPI with 2,6-lutidine from 0 to 5 equivalent at $0.1 \mathrm{Vs}^{-1}$ and the anodic current density as a function of ratio of [2,6-lutidine]/[NHPI]. C) The free energy of the formation of the conjugate acid of NHPI, NHPI/base complex formation. (B3LYP/6$311+\mathrm{G}(\mathrm{d})$ with IERPCM $(\mathrm{MeCN}))$.

At scan rates $\leqslant 0.01 \mathrm{Vs}^{-1}$, the voltammetric response became sigmoidal, attaining a steady-state current density (Figure 2C) that suggested redox active species were being generated at the electrode by follow-up reactions in solution. A steady-state current density with the same magnitude was observed consistently for bases with $\mathrm{p} K_{\mathrm{a}}$ values < 15 and was insensitive to base concentration. (SI)

Voltammetric measurements were performed with anodically activated glassy carbon ${ }^{16}$ to examine the possiblecontribution of inner-sphere effects in the apparent redox responses. However, this deliberate change in electrode surface chemistry did not impact the primary voltammetric features, as the observed peak splitting was invariant with the nature of the glassy carbon surface (SI).

NHPI-Base Complex. Identification of any possible reactions between NHPI and base was performed first, as most of PINO-catalyzed oxidations were operated in the presence of bases. When one equivalent of 2,6-lutidine was used as base, a quasi-reversible voltammogram was obtained with $E_{1 / 2}=+0.39 \mathrm{~V}$ vs $E^{0}\left(\mathrm{Fc}^{+} / \mathrm{Fc}\right.$ ) (Figure 2A). The presence of base decreased the standard potential and increased the reversability of NHPI oxidation. The reported $\mathrm{p} K$ a values of NHPI (23.5) ${ }^{11}$ and the 2,6-lutidinium ion (14.1) ${ }^{17}$ in $\mathrm{MeCN}$ suggest that proton exchange is highly unfavourable. Nevertheless, the plausitibility of a hydrogen bond formation between NHPI and pyridine derivative was assessed through IR spectroscopy. NHPI in MeCN shows a characteristic broad $\mathrm{O}-\mathrm{H}$ stretch at $3210 \mathrm{~cm}^{-1}$ (Figure 3A). Addition of 2,6-lutidine caused a decrease in this absorption and the appearance of a very broad absorption at $\sim 2450 \mathrm{~cm}^{-1}$, corresponding to the $\mathrm{O}-\mathrm{H}$ stretch of the hydrogen-bonded complex. ${ }^{18}$ The cumulative spectra were consistent with an equilibrium constant $K_{\text {eq }}=11.8 \mathrm{M}^{-1}$ for a hydrogen-bonded complex (Figure 3A).

To determine the stoichiometry of the hydrogen-bonded complex, cyclic voltammetric titration experiments were conducted at $0.1 \mathrm{Vs}^{-1}$ (Figure 3B). A solution of NHPI in $\mathrm{MeCN}$ was titrated with 2,6-lutidine. The quasi-reversible voltammetric response at $\sim+0.4 \mathrm{~V}$ vs $E^{0}\left(\mathrm{Fc}^{+} / \mathrm{Fc}\right)$ corresponding to the oxidation event of NHPI-lutidine complex grew in magnitude as the concentration of 2,6-lutidine increased, with a concomitant disappearance of the voltammetric response observed in the absence of a base. The anodic peak current density for the oxidation of the complex was saturated until one equivalent of 2,6-lutidine was added, suggesting NHPI and 2,6-lutidine form a 1:1 complex in solution (Figure $3 \mathrm{~B}$ ). The $E_{1 / 2}$ was dependent upon the 
A) Electrochemical oxidation of NHPI with/without bases
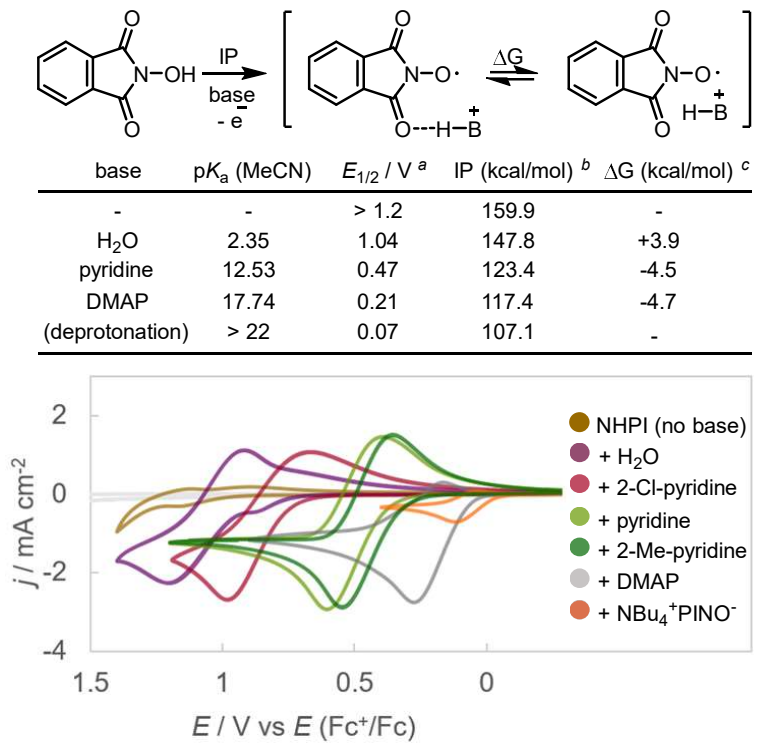

B) Base effect on PINO qeneration and decomposition

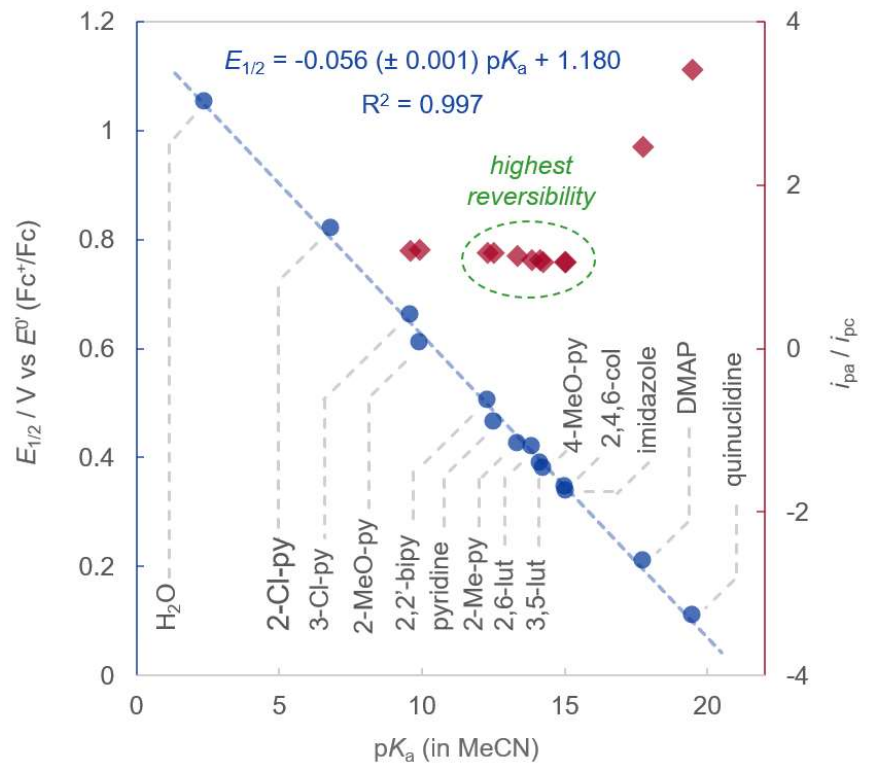

Figure 4. A) Selected cyclic voltammograms and calculations for oxidation of NHPI with/without base. ${ }^{a} E_{1 / 2}$ for oxidation of NHPI in the presence of 1 equivalent of base $0.1 \mathrm{Vs}^{-1} .{ }^{b, c}$ Ionization potential (IP) for oxidation of NHPI complexes, and free energy of dissociation of PINO and the associated conjugate acid. (B3LYP/6-311+G(d) with IEFPCM (MeCN)). B) $E_{1 / 2} \mathrm{vs} \mathrm{p} K_{\mathrm{a}}$ (blue) and $i_{\mathrm{pa}} / i_{\mathrm{pc}}$ vs. $p \mathrm{~K}_{\mathrm{a}}(\mathrm{red})$.

concentration of 2,6-lutidine (SI), consistent with pre-equilibrium complexation.

A set of calculations on the energies for both an ion pair of deprotonated NHPI and pyridinium and for a hydrogenbonded complex was performed (Figure 3C). The free energy change regarding the formation of the 1:1 hydrogenbonded complex between NHPI and pyridine was calculated to be $-2.3 \mathrm{kcal} / \mathrm{mol}$, rendering a formation constant $\left(K_{\mathrm{f}}\right)$ of 47.9. In reasonable agreement with this computation, Abraham's hydrogen-bonding parameters determined for pyridine $\left(\beta_{2}^{H}=0.62\right)^{19}$ and NHPI $\left(\alpha_{2}^{H}=0.37\right)^{20}$ yield an estimate a $K_{\mathrm{f}}$ of 3.9. In contrast, the Brønsted acid-base reaction (i.e. deprotonation) was predicted to be highly endergonic $(\Delta G=+10.6 \mathrm{kcal} / \mathrm{mol})$. With 4-dimethylamino-pyridine (DMAP), deprotonation was expectedly more favourable compared to pyridine, but still calculated to be endergonic $(\Delta G=+1.9 \mathrm{kcal} / \mathrm{mol})$. Similarly, the formation of a NHPIDMAP H-bonded complex is more thermodynamically favoured $(\Delta G=-4.1 \mathrm{kcal} / \mathrm{mol})$ than its NHPI-pyridine counterpart. The smaller $\Delta G$ between full deprotonation and NHPI-DMAP formation $(+6.0 \mathrm{kcal} / \mathrm{mol})$ is consistent with the higher $\mathrm{p} K_{\mathrm{a}}$ of DMAP's conjugate acid (17.7). ${ }^{17}$

Base Effect. To further probe the role of base in PINO generation and decomposition, a series of experiments were carried out with organic bases whose conjugated acids have well-defined $\mathrm{p} K_{\mathrm{a}}$ values in MeCN. ${ }^{17}$ Cyclic voltammograms for the oxidation of NHPI in the presence of one equivalent of different organic bases were collected at $0.1 \mathrm{Vs}^{-1}$ (selected examples presented in Figure 4A, the others in SI). In the presence of stronger bases, NHPI was oxidized at less positive potentials. The dependence of the $E_{1 / 2}$ values on $\mathrm{p} K_{\mathrm{a}}$ are summarized in Figure 4B (blue plots). Across a $\mathrm{p} K_{\mathrm{a}}$ range of 2 to 19 , the linear least squares fit yielded a slope of $56 \pm 1$ $\mathrm{mV}$ per $\mathrm{p} K_{\mathrm{a}}$ unit, consistent with the Nernst factor $\left(2.303 \times \mathrm{RT} / \mathrm{nF}=59 \mathrm{mV} / \mathrm{p} K_{\mathrm{a}}\right)$ for proton-coupled electron transfer (PCET). ${ }^{11}$

A separate analysis of the apparent electron transfer rate constant $\left(k^{0}\right)$ for each voltammogram in Figure S7 did not yield an obvious trend with $\mathrm{p} K_{\mathrm{a}}$. A likely factor was the differences in steric crowding around the pyridine nitrogen atom. For example, voltammetry with bipyridine yielded a much smaller $k^{0}$ value in comparison to pyridine, despite both voltammograms showing similar $E_{1 / 2}$.

Besides the potential shift, we also observed that the magnitude of the cathodic peak current varied with base strength (Figure 4A). Across these organic bases, the ratio of the anodic and cathodic peak current $\left(i_{\mathrm{pa}} / i_{\mathrm{pc}}\right)$ was a function of the $\mathrm{p} K_{\mathrm{a}}$ of the base, approaching 1 as the $\mathrm{p} K_{\mathrm{a}}$ increased to 15 and then increasing at larger $\mathrm{p} K_{\mathrm{a}}$ values (Figure $4 \mathrm{~B}$, red plots). In the presence of stronger bases, e.g. DMAP and quinuclidine, the oxidation was irreversible. While the role of base as a proton acceptor is generally recognized, ${ }^{4-8,11}$ the reason for the erosion of the reversibility of the electrochemical oxidation is not immediately apparent. Based on the lack of reversability above a certain range of $\mathrm{p} K_{\mathrm{a}}$, it would seem that at least under electrochemical conditions, the decomposition of PINO is accelerated under increasingly basic conditions.

In an effort to delineate the effects of individual bases, the redox response of the conjugate base of NHPI (i.e., PINO-) was determined. An NHPI salt, tetrabutylammonium phthalimide- $N$-oxide, was prepared ${ }^{21}$ and its cyclic voltammogram was collected at $0.1 \mathrm{Vs}^{-1}$ in MeCN. Taking $E_{1 / 2}$ to be diagnostic of the formal potential, these data implied $E^{0}$

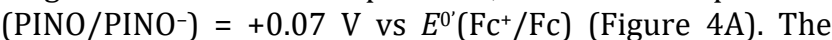
small cathodic peak further suggested PINO itself is unstable on the timescale of the voltammetry. ${ }^{22}$ Based on the cumulative $E_{1 / 2} \mathrm{vs} \mathrm{p} K_{\mathrm{a}}$ data, the voltammetry indicate a $\mathrm{p} K_{\mathrm{a}}$ of $\sim 19$ for NHPI in MeCN. 


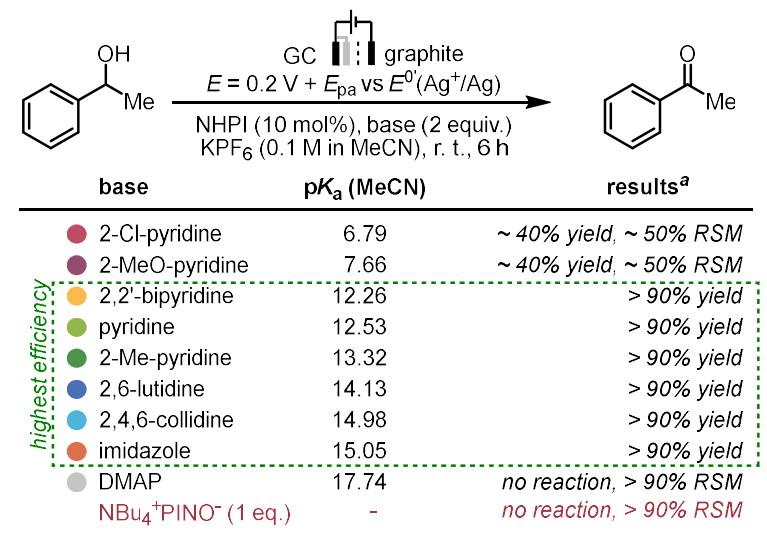

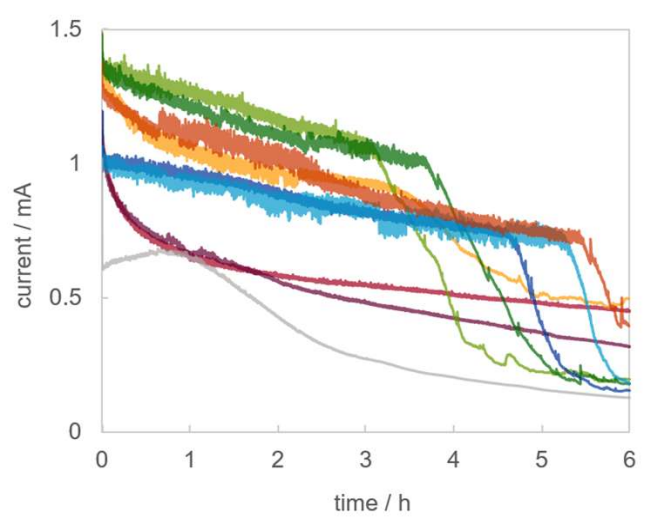

Figure 5. A) Electrolysis conditions: 1 -phenylethanol ( $0.16 \mathrm{mmol})$, NHPI (10 mol\%, $0.016 \mathrm{mmol})$, base ( 2 equiv., $0.32 \mathrm{mmol}), \mathrm{KPF} 6$ $(0.1 \mathrm{M}$ in $8 \mathrm{~mL} \mathrm{MeCN})$, open-air, divided cell, glassy carbon plate anode $\left(2 \mathrm{~cm}^{2}\right)$, graphite cathode $\left(4 \mathrm{~cm}^{2}\right)$, potential controlled electrolysis ( $0.2 \mathrm{~V}$ more positive than anodic peak potential for each redox couple). ${ }^{a}$ Yields determined by GC-MS. B) Chronoamperometry recorded by potentiostat.

For additional insight on the effect of base on electrochemical oxidation, we expanded the computations in Figure $3 \mathrm{C}$ to compare oxidation of NHPI, the H-bonded complexes thereof, and PINO- (Figure 4A). Exepctedly, NHPI was the most difficult species to oxidize, followed by the hydrogen-bonded complexes, and finally PINO-. Importantly, the H-bonded complexes of NHPI and either pyridine or DMAP were predicted to decrease the ionization potential by $24.4 \mathrm{kcal} / \mathrm{mol}$ and $30.4 \mathrm{kcal} / \mathrm{mol}$, respectively relative to the NHPI-water complex. The decrease in ionization potential (IP) parallels the trend of decreasing standard potential for oxidation of NHPI observed experimentally, though a lack of quantitative agreement is not surprising given the absence of implicit solvation, which is presumably particularly relevant in the case of water. Notably, the complexes resulting from oxidation (i.e., PINO---H-base ${ }^{+}$) were found to favor dissociation $(\Delta G=-4.5$ and $-4.7 \mathrm{kcal} / \mathrm{mol}$, respectively), rendering pyridinium or dimethylaminopyridinium and free PINO. Hence, the increased reversibility of NHPI oxidation in the presence of pyridine does not appear to be due to product complexes which increase the persistence of PINO.

A test substrate, 1-phenylethanol, was used to assess whether the base affects the catalytic efficiency. A catalytic current enhancement was observed when the substrate was added to a solution of NHPI and organic bases $\left(\mathrm{p} K_{\mathrm{a}}<\right.$ 15) (SI). The catalytic current was independent of the $p K_{a}$ of the base (SI). Importantly, no current enhancement was observed when stronger bases (i.e., DMAP, NEt3 or quinuclidine) were used. Comparing the catalytic currents measured for 1-phenylethanol and 1-phenylethanol- $d_{1}$ yielded a kinetic isotope effect of 1.8, suggesting that the abstraction of the benzylic hydrogen atom is rate-determining in the electrochemical oxidation (SI).

Electrolysis of 1-phenylethanol catalyzed by NHPI were performed in the presence of different bases at a constant potential for 6 hours (Figure 5). The potential was set +0.2 $\mathrm{V}$ relative to the anodic peak potential for each redox couple. The resulting solutions were analyzed by gas chromatography coupled to a mass spectrometer (GC-MS). The best catalytic efficiency was observed for bases with a $\mathrm{p} K_{\mathrm{a}}$ within the range of $\sim 12$ to 15 , with full conversion within the 6 hours. A substituent effect was noted, as the current magnitude and time to reach full conversion was dependent upon the steric bulk of the base. The less sterically hindered bases promoted higher currents and shorter reaction times. The correlation of reaction time and steric hinderance was in line with the following premise: Weaker bases $\left(\mathrm{p} K_{\mathrm{a}}<10\right)$ displayed a current drop during the reaction and low conversions were obtained. On the other hand, stronger bases $\left(\mathrm{p} K_{\mathrm{a}}>17\right)$ were ineffective for catalysis, resulting in no desired oxidation. Consistent with the foregoing, electrolysis of 1-phenylethanol in the presence of 1 equivalent of the NHPI salt resulted in $>90 \%$ recovery of the starting material.

Mechanistic Insights on PINO decomposition. To understand the causes of catalyst decomposition in order to develop more efficient catalytic systems, PINO decomposition was

Path a. Disproportionation (bimolecular decay)

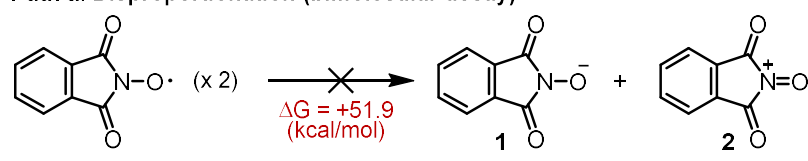

Path b. C-N bond fragmentation (unimolecular decay)
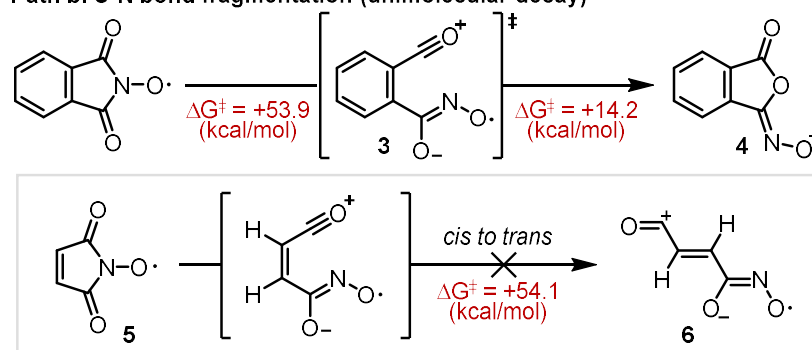

Path c. Retro-Cheletropic reaction (unimolecular decay)

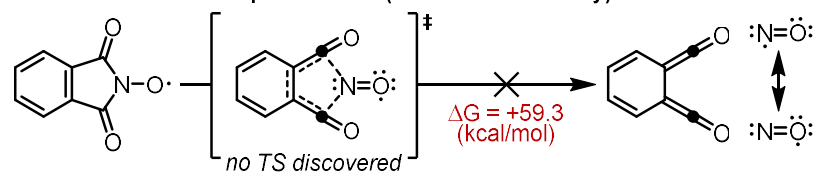

Figure 6. Reaction pathways initially considered for decay of PINO. (B3LYP/6-311+G(d) with IEFPCM (MeCN)). 


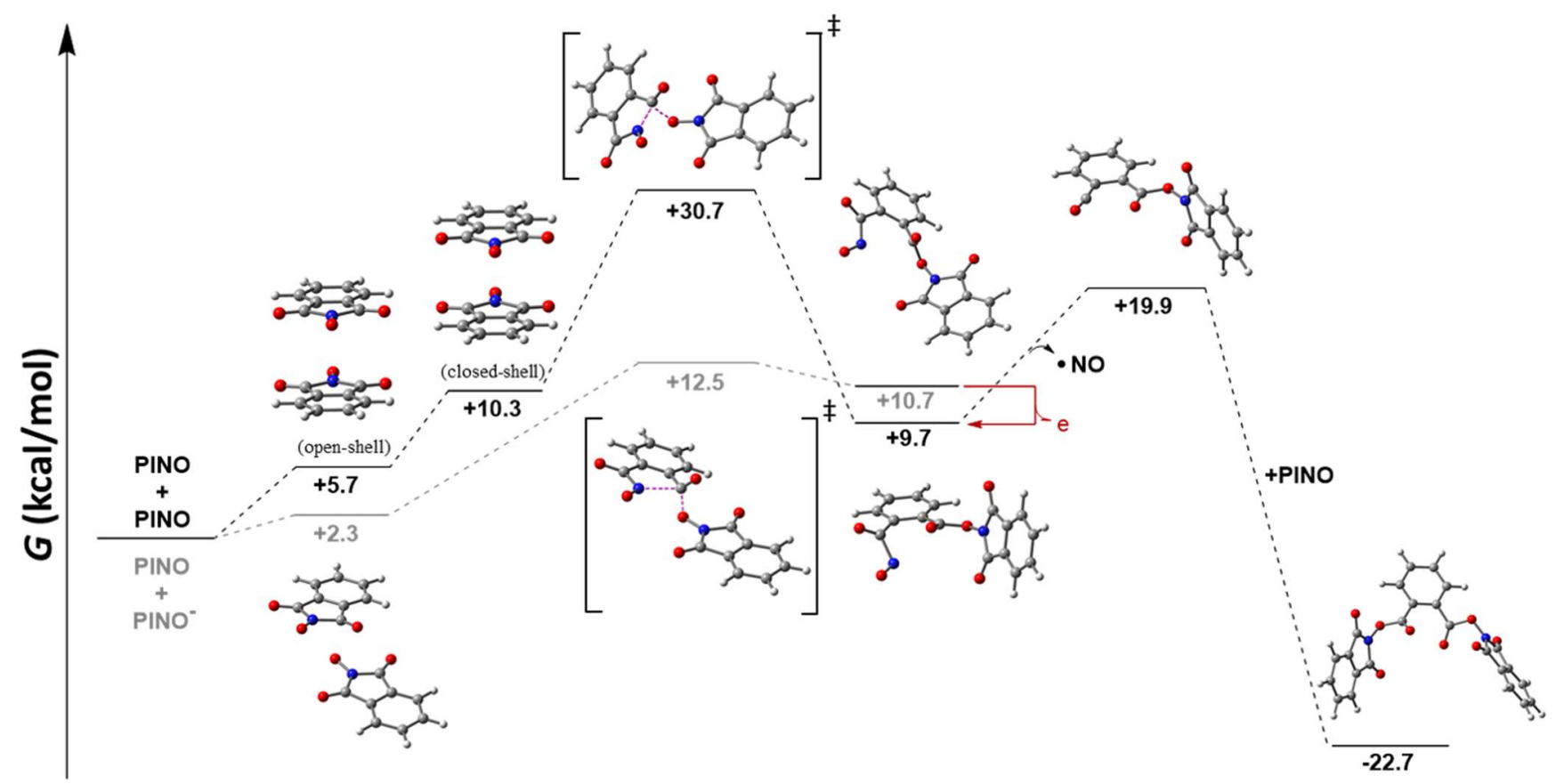

Figure 7. Free energy diagram of proposed bimolecular decomposition pathways of PINO. (B3LYP/6-311+G(d) with IEFPCM (MeCN) and GD3 dispersion correction).

investigated computationally to interrogate both the existing mechanistic proposals as well as others which we considered plausible. Initially, we examined the second-order decay originally proposed by Masui (Figure 1C, path a). ${ }^{9}$ This decomposition path proceeds first by disproportionation of two molecules of PINO as the (presumed) rate determining step. The resulting oxoammonium $\mathrm{PINO}^{+}(2)$ could then undergo acyl substitution by either NHPI or its conjugate base PINO- to yield the putative dimeric intermediate, which could subsequently lead to the isolated trimer (vide infra). Unlike the oxoammonium ion derived from TEMPO, $\mathrm{PINO}^{+}$has not yet been reported in the literature. Indeed, the computed free energy difference between two molecules of PINO and PINO- $+\mathrm{PINO}^{+}(\Delta G=+51.9 \mathrm{kcal} / \mathrm{mol}$, Figure 6 , path a) suggests that disproportionation is highly endergonic. Furthermore, the IP of PINO was computed to be highly similar to that of NHPI $(\Delta G=+158.6 \mathrm{vs}+159.9$ $\mathrm{kcal} / \mathrm{mol}$ ), suggesting that the potential required for anodic oxidation would be well beyond the potential at which oxidation of the NHPI-base complex occurs. On this basis, the formation of a discrete intermediate $\mathbf{2}$, as originally depicted by Masui, is unlikely to occur.

The previously suggested $\mathrm{C}-\mathrm{N}$ fragmentation was also examined (Figure $1 \mathrm{C}$, path b). ${ }^{10}$ The only productive mode of $\mathrm{C}-\mathrm{N}$ cleavage identified by computational means was through the rotation of one of the C(aryl)-C(acyl) bonds (Figure 6, path a), which led to a high energy charge-separated activated complex $\mathbf{3}$ with acylium cation and amide anion $N$-oxyl moieties $(\Delta G=+53.9 \mathrm{kcal} / \mathrm{mol})-$ not an acyl radical. Intrinsic reaction coordinate (IRC) calculations revealed that this transition state collapses to phthalic anhydride monoimine- $N$-oxyl 4, obviously a consequence of the conformational restriction imposed on the reactive moieties by the aryl ring to which they are attached. To provide some insight on the intrinsic strength of the $\mathrm{C}-\mathrm{N}$ bond in question, we determined the energy difference between maleimide- $N$-oxyl 5 and the $(E)$-isomer of its $\mathrm{C}-\mathrm{N}$ bond fragmentation product 6 (Figure 6, path $\mathrm{b}$ ). This transformation has essentially the same free energy cost as formation of the activated complex of PINO heterolysis $(\Delta G=+54.1$ $\mathrm{kcal} / \mathrm{mol}$ ). The results imply that the suggested $\mathrm{C}-\mathrm{N}$ bond cleavage is also unlikely to occur under relevant conditions. In addition, the concerted fragmentation of both $\mathrm{C}-\mathrm{N}$ bonds analogous to the reverse reaction of quinone dimethidebased nitric oxide cheletropic traps (NOCTs) was considered (Figure 6, path c). ${ }^{23}$ Although a transition state was not found, considering the endergonicity of this reaction $(\Delta G=$ $+59.3 \mathrm{kcal} / \mathrm{mol}$ ), it is also unlikely to contribute to the decay of PINO. Admittedly, this point is perhaps not surprising given NOCT reactions have not been reported as being reversible.

Considering the modes by which two molecules of PINO may dimerize, a reaction pathway similar to Masui's original disproportionation proposal was found (Figure 7). An activated complex corresponding to the combination of PINOand $\mathrm{PINO}^{+}$was identified with $\Delta G=+36.5 \mathrm{kcal} / \mathrm{mol}$ relative to two isolated PINO molecules - much lower in energy than the sum computed from the energies of the discrete PINOand $\mathrm{PINO}^{+}$ions $(\Delta G=+51.9 \mathrm{kcal} / \mathrm{mol}$, Figure $6 \mathrm{~A})$. IRC calculations suggest that this transition state connects Masui's aforementioned dimeric intermediate to a PINO contact ion pair at $\Delta G=+16.8 \mathrm{kcal} / \mathrm{mol}$ relative to two isolated PINO molecules. It seems reasonable to suggest that this contact ion pair results from charge transfer from a corresponding PINO radical pair to avoid formation of the higher energy separated $\mathrm{PINO}^{-}$and $\mathrm{PINO}^{+}$ions. Indeed, the corresponding 

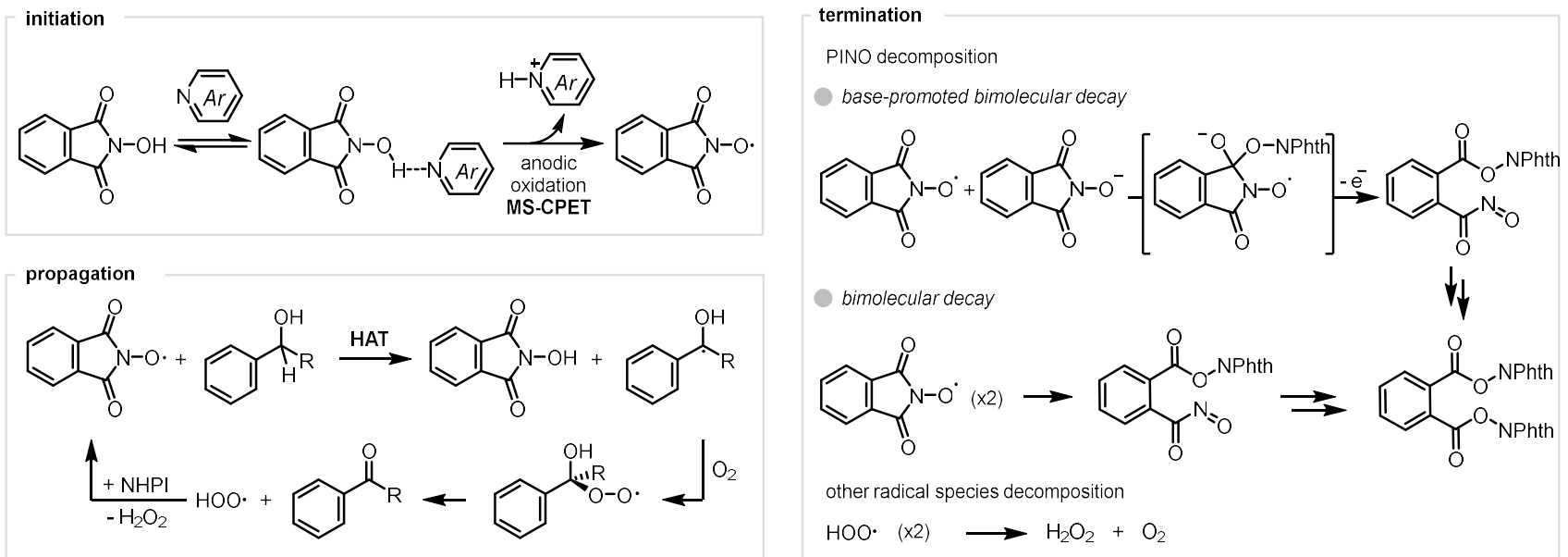

Figure 8. Mechanisms of the electrochemical generation and decomposition of PINO.

PINO (singlet) radical pair is $7.0 \mathrm{kcal} / \mathrm{mol}$ lower in free energy than the contact ion pair. Suspecting that the free energy difference between the PINO radical pair and two separated PINO radicals ( $9.8 \mathrm{kcal} / \mathrm{mol})$ was overestimated on account of DFT's failure to account for dispersion interactions, we incorporated Grimme's empirical dispersion correction (GD3) ${ }^{24}$ in our calculations. Doing so led to a decrease in the free energy change for association of the PINO radical pair (to $+5.7 \mathrm{kcal} / \mathrm{mol}$ ), the contact ion pair (to +10.3 $\mathrm{kcal} / \mathrm{mol}$ ) and overall barrier for substitution (to +30.7 $\mathrm{kcal} / \mathrm{mol}$ ) - see Supporting Information for further details.

An additional bimolecular pathway involving acyl substitution was assessed. Given the the lack of reversibility observed when the tetrabutylammonium salt of NHPI is oxidized or when stronger bases are present, we wondered if $\mathrm{PINO}^{-}$may attack PINO directly. Indeed, a comparatively low energy transition state structure was readily identified for this substitution (Figure 7). The transition state was characterized by significant $\mathrm{O}-\mathrm{C}$ bond formation and $\mathrm{C}-\mathrm{N}$ bond cleavage. IRC calculations revealed that this structure connected a PINO/PINO- complex and the substitution product independent of a tetrahedral intermediate. Again, inclusion of the GD3 empirical dispersion correction systematically decreased the overall computed free-energy barrier from +17.9 to $+12.5 \mathrm{kcal} / \mathrm{mol}$. Although, as in the case of the reaction of two PINO molecules, the reaction is endergonic $(\Delta G=+10.7 \mathrm{kcal} / \mathrm{mol})$, the resultant amide anion $N$-oxyl should oxidize readily since its ionization potential is essentially equivalent to that calculated for PINO- $^{-}(\Delta G$ $=106.3$ vs $107.1 \mathrm{kcal} / \mathrm{mol}$, respectively). ${ }^{25}$ This oxidation would generate Masui's proposed dimeric intermediate.

The optimized structure of Masui's proposed dimer was characterized by a rather conspicuously long $\mathrm{C}-\mathrm{N}$ bond between the carbonyl carbon and nitroso nitrogen atoms (1.59 A), implying it to be quite weak. Indeed, the calculated bond dissociation free energy (BDFE) is $+10.2 \mathrm{kcal} / \mathrm{mol}$, implying the homolysis would occur readily under the reaction conditions, releasing nitric oxide and yielding the corresponding acyl radical. The incipient acyl radical could be trapped by PINO, in a reaction that is predicted to be 42.6 $\mathrm{kcal} / \mathrm{mol}$ downhill. ${ }^{26}$ It should be noted that, in absolute terms, the B3LYP/6-311+G(d) method used for the foregoing calculations is likely to underestimate these bond strengths. Therefore to provide further insight, we carried out high accuracy CBS-QB3 calculations on a model model wherein the 'spectator' PINO moiety is replaced with a hydroxyl group, which yielded corresponding free energies of +15.1 and $-58.7 \mathrm{kcal} / \mathrm{mol}$, respectively). ${ }^{27}$ Nevertheless, the results support a transitory 'dimeric' intermediate and a stable 'trimeric' product in line with the observational evidence accrued to date.

Considering the PINO decomposition mechanism is base promoted, the decomposition rate is base dependent. A series of low scan rate cyclic voltammetric measurement was performed at $0.01 \mathrm{Vs}^{-1}$ as in Figure 2c. Among these investigated bases, the steady-state currents were observed consistently (SI). With weak bases $\left(\mathrm{p} K_{\mathrm{a}}<15\right)$, the steady-state current was independent of base strength and base concentration. When stronger bases $\left(\mathrm{p} K_{\mathrm{a}}>15\right)$ were used, the steady-state current was proportional to the base concentration. Taking this information together, we posit that the observed steady state current indicated that PINO decomposition produces a new redox active species that is easier to oxidize. These voltametric observations are in agreement with aforementioned computional studies.

\section{- DISCUSSION}

The presented data speak to the following three points. First, PINO-catalyzed electrochemical oxidation occurs by a MS-CPET mechanism. Second, the reversibility for electrochemical oxidation of NHPI is dependent on the base strength, and consequently the base strength affects the conversion efficiency of the electrocatalytic oxidation of 1phenylethanol. Third, PINO decomposition is promoted by base via nucleophilic substitution by $\mathrm{PINO}^{-}$on a carbonyl of a second PINO followed by single electron oxidation of the resultant complex.

The linear dependence of $E_{1 / 2}$ on $\mathrm{p} K_{\mathrm{a}}$ with a slope of $56 \pm 1$ $\mathrm{mV} / \mathrm{p} K_{\mathrm{a}}$, observed for the oxidation of NHPI in the presence of base, is a hallmark of PCET reactions. ${ }^{11}$ Indeed, Mayer and co-workers have suggested MS-CPET as a plausible mechanism for the base-mediated formation of PINO from NHPI, in analogy to their study on base mediated oxidation of $\mathrm{N}$ - 
hydroxy-2,2,6,6-tetramethyl-piperidine (TEMPO-H). ${ }^{18}$ The $E_{1 / 2}$ vs $\mathrm{p} K_{\mathrm{a}}$ correlation addresses two further aspects. First, these data indicate that $E^{0^{\prime}}\left(\mathrm{NHPI}^{\bullet+} / \mathrm{NHPI}\right)$ is $\geq+1.05 \mathrm{~V}$ vs $E^{0^{\prime}}\left(\mathrm{Fc}^{+} / \mathrm{Fc}\right)$, in agreement with prior predictions from theory. ${ }^{11}$ Second, these data indicate that $E^{0^{\prime}}$ (PINO/PINO-) is closer to $+0.07 \mathrm{~V}$ vs $E^{0^{\prime}}\left(\mathrm{Fc}^{+} / \mathrm{Fc}\right)$ - which is noticeably more positive than prior theoretical predictions of $E^{0}(\mathrm{PINO} /$ $\left.\mathrm{PINO}^{-}\right)=-0.1 \mathrm{~V}$ vs $E^{0^{\prime}}\left(\mathrm{Fc}^{+} / \mathrm{Fc}\right) .{ }^{11}$ Similarly, the cumulative voltammetric measurements shown here indicate that the $\mathrm{p} K_{\mathrm{a}}$ of NHPI in MeCN is 19 (rather than 23.5). ${ }^{11}$

The subject of PINO decomposition has been a matter of interest since the seminal kinetic studies conducted by Masui, ${ }^{9}$ although the details have remained hitherto nebulous. Both first ${ }^{28}$ and second order decay, ${ }^{9,29}$ with respect to PINO, have been reported leading to a bifurcation of proposed decay processes that have been presumed to lead to the same trimer decomposition product. ${ }^{8}$ This paper argues that ascribing the observed first-order decay ${ }^{28}$ of PINO to a unimolecular phenomenon perhaps takes too much for granted. The underlying assumption is that the principal product of the process is the same trimer previously observed by Masui. Additionally, as pointed out by Pedulli, the kinetics of decay are quite dependent on solvent composition, ${ }^{28 a}$ an observation which seems inconsistent with unimolecular decay being rate-determining. However, without more information on the actual product(s) formed as a result of the first-order decay process(es), or the prerequisites for its occurrence, searching for the rate defining reaction in silico may be futile. Suffice to say, the oft-invoked fragmentation of the $\mathrm{C}-\mathrm{N}$ bond in PINO to form a transient acyl radical which can be captured appears unlikely.

The presented data suggest that the strength of the base alters the catalytic efficiency by influencing PINO decomposition. The voltammetric measurements and electrolysis experiments suggest an optimal base strength in the $\mathrm{p} K_{\mathrm{a}}$ range of $\sim 12-15$. Specifically, we posit that PINO has two operative decomposition pathways in MeCN (Figure 7 and 8). One decomposition involves the dimerization of two molecules of PINO via a charge-transfer complex. In the presence of weak bases $\left(\mathrm{p} K_{\mathrm{a}}<15\right)$, this pathway is dominant. In the presence of comparatively strong bases $\left(\mathrm{p} K_{\mathrm{a}}>15\right)$, a second significantly faster decomposition pathway consisting of the reaction between $\mathrm{PINO}^{-}$and PINO is operative. The resulting intermediate is rapidly oxidized at the anode to produce the same dimer. This oxidation event is observable at the slow scan rate voltammetry. The dimer undergoes C-N cleavage to release nitric oxide and form an acyl radical which reacts with another molecule of PINO to form trimer.

Our expansion on early observations regarding base-promoted oxidations and elucidation of a reasonable PINO decomposition mechanism provides guidance to advance PINO-catalyzed electrochemical oxidations. Previous efforts on designing PINO-derived HAT catalysts have largely focused on adjusting BDFEs to improve reactivity. In contrast, an approach to improving catalysis through extension of the effective catalytic lifetime of PINO by obviating degradation pathways has not been a focus of research efforts. On the basis of the results presented herein, we suggest that both of these aspects must be considered in the design of more effective $\mathrm{N}$-oxyl catalysts for $\mathrm{C}$-H oxidation reactions.
In summary, we have described a comprehensive electroanalytical study of the NHPI/PINO redox mediator, and conducted computational studies to uncover the mechanisms by which PINO degrades as a function of the identity of the base. These data provide a road map for developing more effective $\mathrm{N}$-oxyl catalysts for $\mathrm{C}$-H oxidation reactions.

\section{- ASSOCIATED CONTENT}

\section{Supporting Information}

The Supporting Information is available free of charge on the ACS Publications website at [ACS WEBSITE].

Supplementary materials including cyclic voltammetry, electrolysis, compound characterization and calculations. (PDF)

\section{- AUTHOR INFORMATION}

\section{Corresponding Authors}

Corey R. J. Stephenson - Department of Chemistry, University of Michigan, Ann Arbor, Michigan 48109, United States; OCRID: 0000-0002-2443-5514; Email: crjsteph@umich.edu

Stephen Maldonado - Department of Chemistry, and Program in Applied Physics, University of Michigan, Ann Arbor, Michigan 48109, United States; OCRID: 0000-0002-29174851; Email: smald@umich.edu

Derek A. Pratt - Department of Chemistry and Biomolecular Sciences, University of Ottawa, Ottawa, Canada K1N6N5; OCRID: 0000-0002-7305-745X; Email: dpratt@uottawa.ca

\section{Authors}

Cheng Yang - Department of Chemistry, University of Michigan, Ann Arbor, Michigan 48109, United States; OCRID: 00000001-7929-2349.

Luke A. Farmer - Department of Chemistry and Biomolecular Sciences, University of Ottawa, Ottawa, Canada K1N6N5.

\section{Notes}

The authors declare no competing financial interest.

\section{- ACKNOWLEDGMENT}

C.R.J.S. acknowledges the financial support from the National Science Foundation (CHE-1565782 and CBET-2033714), the Camille and Henry Dreyfus Foundation, and the University of Michigan. S.M. acknowledges the generous support from the Department of Energy (DE-SC0006628). D.A.P. acknowledges the financial support from the Natural Sciences and Engineering Research Council of Canada. This work was enabled by support provided by Compute Ontario and Compute Canada (www.computecanada.ca).

\section{- REFERENCES}

(1) Yan, M.; Kawamata, Y; Baran, P. S. Synthetic Organic Electrochemical Methods Since 2000: On the Verge of a Renaissance. Chem. Rev. 2017, 117, 13230-13319.

(2) Moeller, K. D. Synthetic Applications of Anodic Electrochemistry. Tetrahedron 2000, 56, 9527-9554.

(3) Nutting, J. E.; Rafiee, M.; Stahl, S. S. Tetramethylpiperidine $N$ Oxyl (TEMPO), Phthalimide $N$-Oxyl (PINO), and Related $N$-Oxyl Species: Electrochemical Properties and Their Use in Electrocatalytic Reactions. Chem. Rev. 2018, 118, 4834-4885. 
(4) Horn, E. J.; Rosen, B. R.; Chen, Y.; Tang, J.; Chen, K.; Eastgate, M. D.; Baran, P. S. Scalable and Sustainable Electrochemical Allylic C-H Oxidation. Nature 2016, 533, 78-81.

(5) Masui, M.; Ueshima, T.; Ozaki, S. $N$-Hydroxyphthalimide as an Effective Mediator for the Oxidation of Alcohols by Electrolysis. Chem. Commun. 1983, 479-480.

(6) Bosque, I.; Magallanes, G.; Rigoulet, M.; Kärkäs, M. D.; Stephenson, C. R. J. Redox Catalysis Facilitates Lignin Depolymerization. ACS Cent. Sci. 2017, 3, 621-628.

(7) Rafiee, M.; Wang, F.; Hruszkewycz, D. P.; Stahl, S. S. N-Hydroxyphthalimide-Mediated Electrochemical Iodination of Methylarenes and Comparison to Electron-Transfer-Initiated C-H Functionalization. J. Am. Chem. Soc. 2018, 140, 22-25.

(8) Recupero, F.; Punta, F. Free Radical Functionalization of Organic Compounds Catalyzed by $\mathrm{N}$-Hydroxyphthalimide. Chem. Rev. 2007, 107, 3800-3842.

(9) Ueda, C.; Noyama, M.; Ohmori, H.; Masui, M. Reactivity of Phthalimide- $N$-oxyl: A Kinetic Study. Chem. Pharm. Bull. 1987, 35 1372-1377. Note: In Masui's report, the potential controlled electrolysis was performed at $0.9 \mathrm{~V}$ vs SCE, which is $0.52 \mathrm{~V}$ vs $E^{0}$ $\left(\mathrm{Fc}^{+} / \mathrm{Fc}\right.$ ) (see ref. 15).

(10) Amorati, R.; Lucarini, M; Mugnaini, V.; Pedulli, G. F.; Minisci, F.; Recupero, F.; Fontana, F.; Astolfi, P.; Greci, L. Hydroxylamines as Oxidation Catalysts: Thermochemical and Kinetic Studies. J. Org. Chem. 2003, 68, 1747-1754.

(11) Warren, J. J.; Tronic, T. A.; Mayer, J. M. The Thermochemistry of Proton-Coupled Electron Transfer Reagents and its Implications. Chem. Rev. 2010, 110, 6961-7001. Note: The reported $\mathrm{p} K_{\mathrm{a}}$ of NHPI in MeCN is an extrapolated value based on $\mathrm{p} K_{\mathrm{a}}$ measured in DMSO rather than something directly measured in MeCN. See: (a) Koppel, I.; Koppel, J.; Leito, J.; Pihl, V.; Grehn, L.; Ragnarsson, U. J. Chem. Res. 1993, 11, 3008-3028. (b) Kütt, A.; Leito, I.; Kaljurand, I.; Sooväli, L.; Vlasov, V. M.; Yagupolskii, L. M.; Koppel, I. A. J. Org. Chem. 2006, 71, 2829-2838.

(12) Sawyer, D. T.; Sobkowiak, A.; Roberts, J. L. Electrochemistry for Chemists, 2nd Edition, 1995, Chapter 7.

(13) Kishioka, S.; Yamada, A. Kinetic Study of the Catalytic Oxidation of Benzyl Alcohols by Phthalimide- $N$-Oxyl Radical Electrogenerated in Acetonitrile using Rotating Disk Electrode Voltammetry. J. Electroanal. Chem. 2005, 578, 71-77.

(14) Rafiee, M.; Karimi, B.; Alizadeh, S. Mechanistic Study of the Electrocatalytic Oxidation of Alcohols by TEMPO and NHPI. ChemElectroChem 2014, 1, 455-462.

(15) $0 \mathrm{~V}$ vs $E^{0^{\prime}}\left(\mathrm{Fc}^{+} / \mathrm{Fc}\right)=+0.38 \mathrm{~V}$ vs $\mathrm{SCE}=+0.63 \mathrm{~V}$ vs NHE. See: Pavlishchuk, V. V.; Addison, A. W. Conversion Constants for Redox Potentials Measured Versus Different Reference Electrodes in Acetonitrile Solutions at $25^{\circ} \mathrm{C}$. Inorganica Chimica Acta 2000, 298, 97 102.

(16) Sawant, T. V.; McKone, J. R. Flow Battery Electroanalysis. 2. Influence of Surface Pretreatment on $\mathrm{Fe}^{(\mathrm{III} / \mathrm{II})}$ Redox Chemistry at Carbon Electrodes. J. Phys. Chem. C 2019, 123, 144-152.

(17) a) Lõkov, M.; Tshepelevitsh, S.; Heering, A.; Plieger, P. G.; Vianello, R.; Leito, I. On the Basicity of Conjugated Nitrogen Heterocycles in Different Media. Eur. J. Org. Chem. 2017, 4475-4489. b) Kaljurand, I.; Rodima, T.; Leito, I.; Koppel, I. A.; Schwesinger, R. SelfConsistent Spectrophotometric Basicity Scale in Acetonitrile Covering the Range between Pyridine and DBU. J. Org. Chem. 2000, 65,
6202-6208. c) K, I. M.; Ikeda, S. Polarographic and Acid Properties of Thorium Perchlorate in Acetonitrile. J. Phys. Chem. 1961, 65, 1020-1026. d) http://ibond.nankai.edu.cn.

(18) Morris, W. D.; Mayer, J. M. Separating Proton and Electron Transfer Effects in Three-Component Concerted Proton-Coupled Electron Transfer Reactions. J. Am. Chem. Soc. 2017, 139, 10312 10319.

(19) Abraham, M. H.; Grellier, P. L.; Prior, D. V.; Morris, J. J.; Taylor, P. J. J. Chem. Soc. Perkin Trans. 2. 1990, 521-529.

(20) The $K_{\mathrm{f}}$ of NHPI with MeCN $\left(\beta_{2}^{H}=0.44\right)^{18}$ is reported $(1.3 \mathrm{M}$ $\left.{ }^{1}\right)^{27}$ a from which is $\alpha_{2}^{H}$ calculated $\left(\log K_{\mathrm{f}}=7.354 \alpha_{2}^{H} \beta_{2}^{H}-1.094\right)$.

(21) Dekamin, M. G.; Mokhtari, J.; Naimi-Jamal, M. R. Organocatalytic cyanosilylation of carbonyl compounds by tetrabutylammonium phthalimide- $N$-oxyl. Catalysis Communications 2009, 10, 582-585.

(22) The current magnitude for oxidation of the NHPI salt was smaller than the other NHPI/base complex. This was possibly due to a self-complexation between the ionic species, which was presented in the SI.

(23) Korth, H.-G.; Sustmann, R.; Lommes, P.; Paul, T.; Ernst, A.; de Groot, H.; Hughes, L.; Ingold, K. U. J. Am. Chem. Soc. 1994, 116, 27672777.

(24) Grimme, S.; Antony, J.; Ehrlich, S.; Krieg, H. A Consistent and Accurate $a b$ initio Parameterization of Density Functional Dispersion Correction (DFT-D) for the 94 Elements H-Pu. J. Chem. Phys. 2010, 132, 154104.

(25) Shown is the IP for amide anion $\mathrm{N}$-oxyl without GD3 correction applied, and it was found to have no impact on the computed value regardless (106.3 vs $106.1 \mathrm{kcal} / \mathrm{mol}$ ).

(26) The DFT BDFE values were calculated both with and without GD3 empirical correction for the $\mathrm{C}-\mathrm{N}$ of the dimer complex $(+10.2 /+7.2 \mathrm{kcal} / \mathrm{mol}$ respectively $)$ and the $\mathrm{C}-0$ of the trimer complex $(+42.1 /+38.4 \mathrm{kcal} / \mathrm{mol}$ respectively $)$. The former is highlighted in the main text for direct comparison with the bimolecular pathways described vide supra.

(27) The C-O and C-N BDE/BDFE values were elected to calculate using a high accuracy CBS-QB3 method (gas-phase) with the phthalimide on the other carbonyl truncated to a hydroxyl group. Replacing the phthalimide with hydroxyl was found to have a negligible impact on the BDE/BDFE when using the B3LYP/6$311+\mathrm{G}(\mathrm{d})$ method sans GD3 $(\leq 1.5 \mathrm{kcal} / \mathrm{mol})$. The calculated C-O BDE/BDFE of the model trimer was $71.3 / 58.7 \mathrm{kcal} / \mathrm{mol}$. The calculated C-N BDE/BDFE of the model intermediate dimer was $26.7 / 15.1 \mathrm{kcal} / \mathrm{mol}$. For comparison, $26.7 \mathrm{kcal} / \mathrm{mol}$ is $\sim 10$ $\mathrm{kcal} / \mathrm{mol}$ lower than the 0-O BDE of a typical alkyl peroxide. See: Bach, R. D.; Ayala, P. Y.; Schlegel, H. B. J. Am. Chem. Soc. 1996, 118, 12758-12765.

(28) (a) Amorati, R.; Lucarini, M.; Mugnaini, V.; Pedulli, G. M.; Minisci, F.; Recupero, F.; Fontana, F.; Astolfi, P.; Greci, L. J. Org. Chem. 2003, 68, 1747-1754. (b) Kushch, O.; Hordieieva, I.; Novikova, K.; Litvinov, Y.; Kompanets, M.; Shendrik, A.; Opeida, I.; J. Org. Chem. 2020, 85, 7112-7124.

(29) (a) Koshino N., Saha B., Espenson J. H. J. Org. Chem., 2003 68, 9364-9370. (b) Baciocchi E., Gerini M. F., Lanzalunga O. J. Org. Chem., 2004, 69, 8963-8966. 